\title{
TwisterSearch: A Distributed User Interface for Collaborative Web Search
}

\author{
Roman Rädle, Hans-Christian Jetter, and Harald Reiterer
}

\begin{abstract}
Although a Web search is typically regarded as a solitary activity, collaborative search approaches are becoming an increasingly relevant topic for HCI and distributed user interfaces (DUIs). Today's collaborative search systems lack comprehensive search support that also involves pre- or post-search activities such as preparing for a search or making sense of search results. We believe that post-WIMP DUIs can help to better support social searches and have identified four design goals that are critical for their successful design. In consequence, we present TwisterSearch, an interactive DUI prototype that meets our four design goals. A formative study conducted with students at a high school shows its general applicability for educational purposes.
\end{abstract}

\subsection{Introduction}

In the recent years, research in HCI has increasingly focused on collaborative searches [1-6]. Collaborative search approaches can support activities and decision making such as planning travel, purchasing products, or searching for literature and could become important tools for users' information practice in future. Consequentially, Morris identified a great need for better tool support for collaborative Web searches [7].

We believe that distributed user interfaces (DUI) as defined by Elmqvist [8] are particularly appropriate for supporting collaborative Web searches, especially

R. Rädle $(\bowtie) \cdot H$. Reiterer

Human-Computer Interaction Group, University of Konstanz, Konstanz, Germany

e-mail: roman raedle@uni-konstanz.de; harald reiterer@uni-konstanz.de

H.-C. Jetter

Intel Collaborative Research Institute for Sustainable Connected Cities (ICRI Cities), University College London, London, UK

e-mail: h.jetter@ucl.ac.uk 


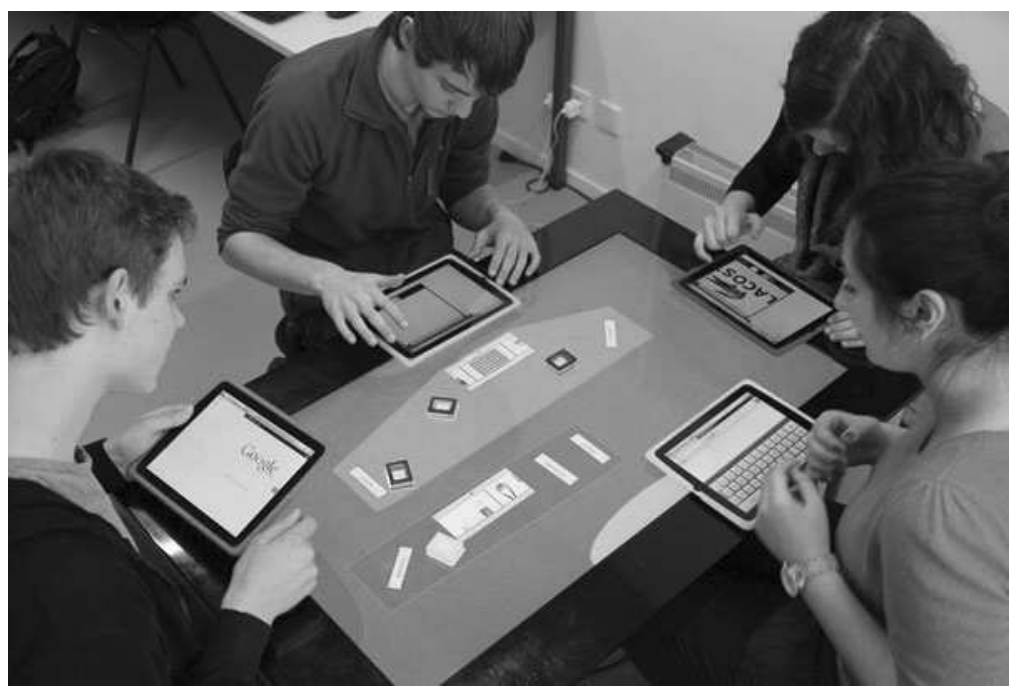

Fig. 5.1 A group of four students performing a Web search with TwisterSearch. Each student was assigned to a red, green, yellow, or blue color with which to identify themselves during the search process. The Apple iPad allows solitary Web searches as well as a seamless transition between different coupling styles without hindering others

when assisting users in the three search phases identified by Evans and Chi in their canonical model of social search based on everyday searches, including before search, during search, and after search [9].

While most present-day systems for collaborative Web searches focus on the during search phase, they lack support for other phases that are more collaborative and they are often distributed in nature and require a division of labor. For example, a survey conducted by Morris showed that $22.0 \%$ of the respondents cooperated by brainstorming or suggesting keywords to each other for generation and refinement purposes before the search [7] - a process that is currently unsupported.

Furthermore, we believe that DUIs based on post-desktop computing systems such as tabletops and tablets are important for a natural collaboration and for supporting different working styles. For example, Jetter et al. provide collaborative faceted search and flexible working styles using a hybrid visual-tangible user interface on a tabletop that users perceived as fun to use and that was equally effective as traditional Web interfaces [4].

Our goal is to achieve a similar result for collaborative Web searches based on a Samsung SUR40 with Microsoft ${ }^{\circledR}$ PixelSense ${ }^{\mathrm{TM}}$ tabletop and Apple iPad tablets. In the following, we first propose design goals for systems supporting collaborative Web searches based on the canonical model of social search by Evans and Chi [9] and implications for design of Morris [7]. Then, we present TwisterSearch ${ }^{1}$ (Fig. 5.1), an interactive prototype that we designed and

${ }^{1}$ TwisterSearch Video - http://hci.uni-konstanz.de/researchprojects/twistersearch 
implemented to meet these design goals, and describe its interaction design using a scenario. Thereafter, a real-life study demonstrates its overall usability for educational purposes. We conclude with a brief summary and our plans for future work.

\subsection{Design Goals}

We have formulated four design goals (DG1-4) based on the canonical model of social search by Evans and Chi [9] and design indications given by Morris [7]: (1). Support Strategic Planning and Coordination, (2). Amplify Collaboration, (3). Intensify Discussion and Simplify User Input, and (4). Facility Traceability of Evidence Files. We consider all four of the DGs to be critical for the successful design of a post-WIMP DUI for collaborative Web searches. Therefore, our prototype TwisterSearch was designed and implemented with regard to these DGs.

\subsubsection{DG1: Support Strategic Planning and Coordination}

Morris describes two search strategies that occur in cooperative search tasks: divideand-conquer and brute force [7]. The first is a coordinated division of labor whereas the latter is uncoordinated and tends to evoke "Google races" or "competitions." These races could duplicate search results and thus increase search effort. Therefore, we argue that providing tool support for explicit coordination and planning of an on-going search leads towards a structured search. Users should be supported in pre-search activities (e.g., framing contexts and refining requirements) and postsearch activities (e.g., organizing and distributing search results). Thereby, framing the context defines and clarifies the boundaries of an intended future search task and establishes informational needs and motives among the group members. Refining the search requirements solidifies informational needs in a step-by-step manner by consulting other sources, such as colleagues. Later, structuring and distribution of search results takes place in $72.0 \%$ of the reported search experiences and is a precondition for embedding searches into real world activities and decision making [9].

\subsubsection{DG2: Amplify Collaboration}

Evans and Chi categorized the during search phase into three different behaviors: navigational, transactional, and informational search [9]. Based on their survey, the latter accounts for more than half $(59.3 \%)$ of the search intentions and includes various steps, from information foraging to sense-making. The informational search behavior, furthermore, features both solitary tasks (e.g., reading and extracting 
information) as well as informational exchange with others. A Web search system, therefore, should best offer a smooth transition between loosely-coupled parallel work and tightly-coupled collaboration similar to [3, 4].

\subsubsection{DG3: Intensify Discussion and Simplify User Input}

Conventional WIMP interfaces with their single point-of-action are inappropriate for creating shareable user interfaces for co-located collaborative work. In these cases, simultaneous user input is indispensable. In contrast, Geyer et al. show the feasibility of a post-WIMP tabletop and tangible user interface combined with digital pen and paper for creative group work [10]. Furthermore, touch interfaces, such as tabletops, allow users to communicate more efficiently with the help of deictic references to create a joint reference and substantiate arguments. Although touch input is the dominant input on tabletops, a study conducted by Morris et al. also discovered issues when using virtual keyboards on tabletops for search term input and propose the integration of physical keyboards instead [5]. We further believe that collaborative Web search systems enable verbal and non-verbal faceto-face communication and more natural gesturing to intensify discussion and to yield superior outcomes. Besides these effects on communication, a simplified user input also allows users to focus on the primary search task instead of being busy with secondary tasks, such as text input.

\subsubsection{DG4: Facilitate Traceability of Evidence Files}

Gathering results and additional information automatically during a search allows users to trace the directions of the search and the keywords used to find the results. Morris [7] writes that "this information helps collaborators understand what techniques have already been tried and how to interpret the authoritativeness or appropriateness of the results." Thus, we consider traceability to be an important aspect, especially if Web searches are carried out over several sessions.

\subsection{System Design}

Based on the four design goals, we designed and implemented our prototype TwisterSearch. TwisterSearch is a distributed user interface running on a shared display and multiple private displays. The shared display is used to collaboratively collect search results in a visual workspace. This workspace is provided on a Samsung SUR40 with Microsoft ${ }^{\circledR}$ PixelSense ${ }^{\mathrm{TM}}$ tabletop providing multi-touch input and additionally recognizes physical objects placed on the surface (tokens). 


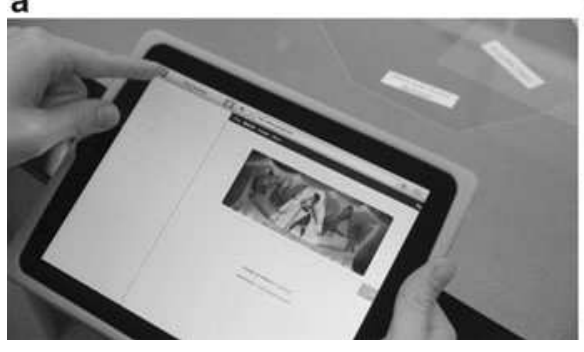

b

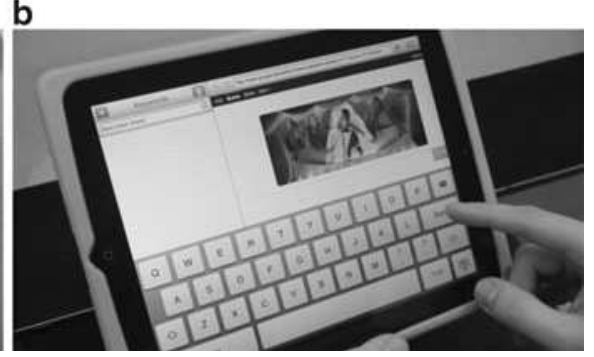

Fig. 5.2 Add new keywords to the search. (a) Tap on the ' + ' sign on the top left. (b) A keyboard appears and you can enter the keyword. Press the 'Done' button on the keyboard to confirm the keyword, which sends it to the shared space automatically

Private pad-sized displays (Apple iPads) are used around the table to individually search the Web and share findings with co-workers on the shared display. The following short scenario provides an example for a typical usage situation.

At the beginning of a history course, four students are requested to do research about the history of Switzerland. They are asked to collect facts about its culture, topology, and politics and write an essay about their findings by the end of the term. They are allowed to do this as a group. The group meets at the library where a workroom is equipped with TwisterSearch. The four students sit around the tabletop and each user takes a TwisterSearch set consisting of an Apple iPad and a small tablet token (see Fig. 5.1). This token is a small acrylic and tablet-shaped glass block with a colored frame. Each set and thus each user has a unique color (red, green, blue, yellow) that is also visible as the color of the iPad's cover and the frame of the tablet token. Before the group members start their individual search activities, they connect their private displays to the shared space by starting the TwisterSearch app on the iPad. A user halo (colored oval) appears at edge of the shared display and indicates that the connection is functioning properly (see Fig. 5.1). Next, they start to frame the topic (DG1). For this purpose, all users type in relevant keywords on their iPads using the virtual keyboard and send the keywords to the shared space by pressing the 'Done' button (see Fig. 5.2) (DG3). Then, keywords are displayed instantly on the shared space in the user's color and close to the user's halo.

Collecting keywords is either done in parallel or as a joint effort in which team members recommend keywords or consult other group members for relevant terms (DG2, DG3). This process leads to a framing of the search's context and results in a collaborative construction of a skeleton of keywords, which is filled with search results in the next step. The keywords appear in the four colors that are each assigned to a single user, which provides a great degree of traceability (DG4). It is possible for one user to start clustering keywords according to their semantic coherence while the others are collecting additional keywords. However, clustering can also be done as a joint effort supported by discussion (DG3).

Clusters are created and become visible when a user encircles one or more keywords using his or her finger. Furthermore, clusters can overlap to convey the search topic (e.g., Switzerland) (Fig. 5.3). Since requirements are often refined in 


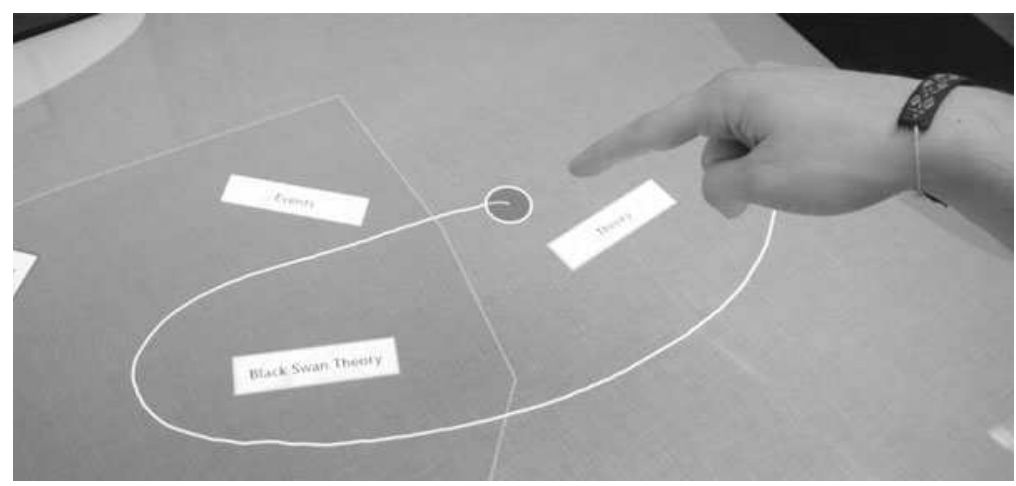

Fig. 5.3 Touch input is used to cluster keywords and to frame the context of the intended informational search
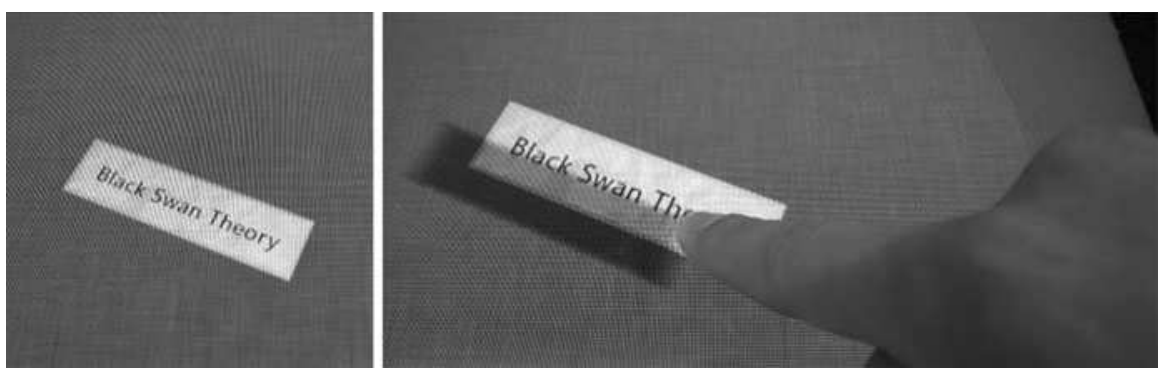

Fig. 5.4 You can lift a keyword by touching and dragging it on the screen. A successful lift will be indicated using a drop shadow effect and a matrix transform

social searches, users can change existing clusters and cluster content at any time. For instance, they can add new keywords, rearrange keywords to different clusters, or split clusters. To lift a keyword virtually, simply has to touch and release it as desired. Lifting and dropping is animated using a drop shadow effect and a matrix transform, which surrogates a behavior similar to moving a real scrap of paper (Fig. 5.4). Moreover, keywords can be removed and put inside the user halo for later usage. After students agree on clustering, the group members are assigned to different clusters by putting the users' corresponding tablet tokens on different clusters (DG1). The token indicates who is responsible for which search so that collaborators know who is searching for information for a specific cluster. This highly increases the group's overall awareness of what each member is doing. Moreover, you can transfer cluster keywords to the linked Apple iPad by placing a token on a cluster.

Now, individual searches are performed on private displays. This can be done either loosely coupled in parallel (no 'backseat driving' [7]) or tightly-coupled in 


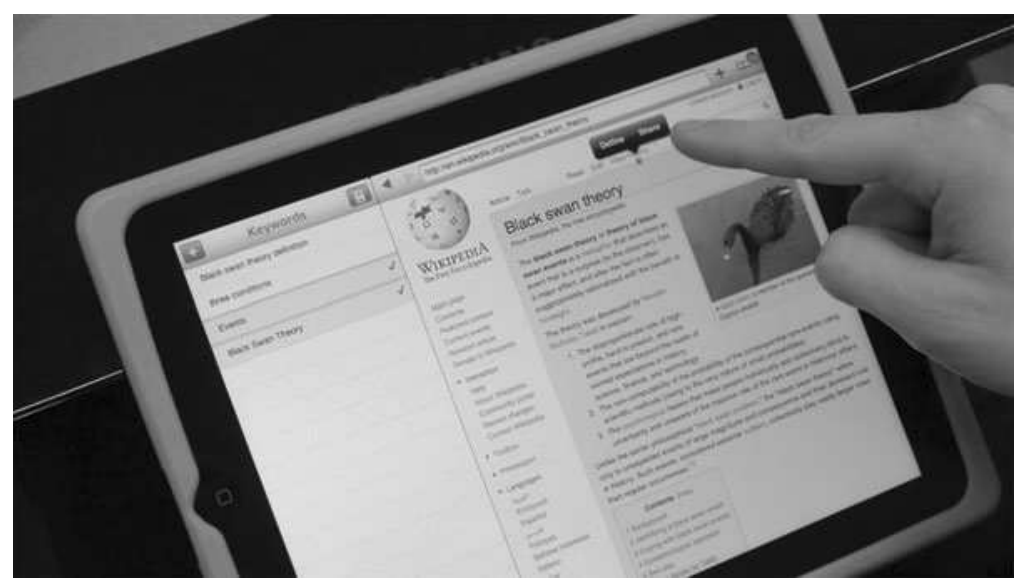

Fig. 5.5 The TwisterSearch user interface on an Apple iPad featuring keywords of a selected cluster (left column) and a Web browser (right column)

collaboration (DG2), such as when showing interesting websites to co-workers. Moreover, novice searchers can learn vocabulary and syntax from experts when searching in close collaboration [7] and apply their knowledge instantly. On the private display, received keywords are displayed in the left column and a Web browser is displayed in the right column (Fig. 5.5). A user selects one or more keywords by tapping on them. Then, a 'Google' search is initiated automatically using the selected keywords and consequently displays 'Google' search results. Users can browse through provided links or adjust the search manually. The browser is operated as known from the Apple iPad Safari app. A complete website or parts of it can be selected by touching and holding the information until the selection rectangle shows up. A 'Share' button then appears above or below the selection. By pressing the 'Share' button, the selected information, including search paths taken and user ID, is transferred to the shared display. The result is displayed immediately in the result view of the cluster on the shared display (Fig. 5.6). Each cluster has its own scrollable result view. Users can hand over private displays to show and exchange interesting information before sharing them with the group. All results can be reviewed on the shared display and private displays at any time. Tapping a result on the shared display opens the corresponding result on all private displays linked through tablet tokens, which is useful for discussion and especially important when defining arguments for the final outcome (DG3).

After a Web search session, results can be automatically stored on any kind of removable disk by connecting it via USB to the Samsung SUR40. The TwisterSearch application creates a dedicated folder on that disk and saves images and Web sites to that folder. This allows the group to distribute or organize results from a collaborative Web search session (DG4). 


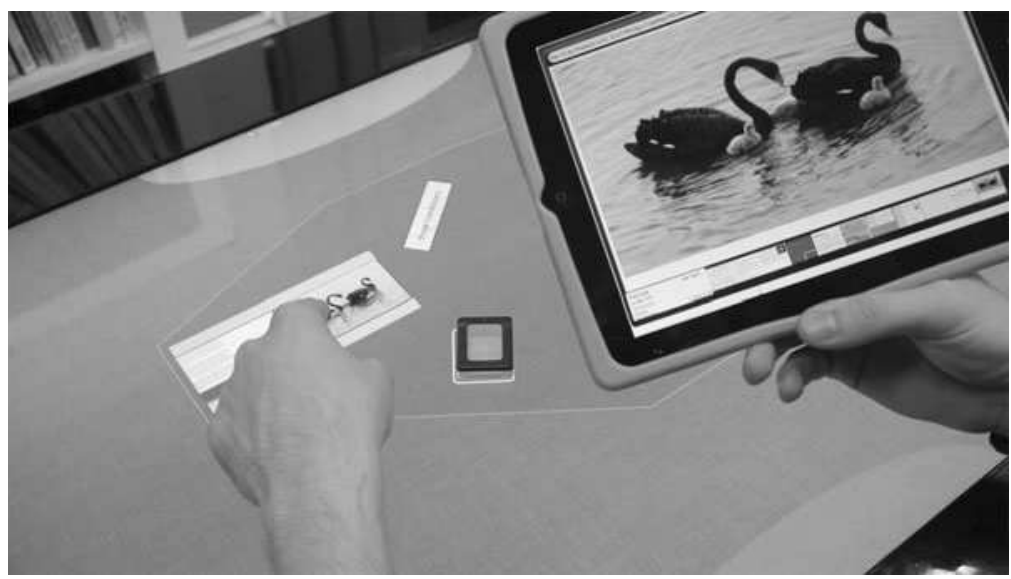

Fig. 5.6 Tapping a result in the result view on the shared display opens the same result in more detail on the private display

\subsection{Implementation}

The application on the shared display (server) is implemented in C\#/WPF with the .NET 4.0 framework and the application on the private display (client) is implemented in iOS 5. The cluster visualization on the Samsung SUR40 displays a convex hull to indicate encircled objects. A Windows Communication Foundation (WCF) Web service and $\mathrm{OSC}^{2}$ is used for client/server communication. The shared display renders Web content with help of Awesomium. ${ }^{3}$ The clients communicate with the server via WCF Web service, which will be opened on the Samsung SUR40 on application startup. Multicast OSC messages distribute keywords and the object IDs of existing results to the clients. Theoretically, the implementation of TwisterSearch supports unlimited clients; however, the table size constrains the number of collaborators to a maximum of four. $\mathrm{BaseX}^{4}$ persists session data, all results including search paths and the user ID, all connection data, and the interaction $\log$. The latter will be used to evaluate the system in a controlled experiment.

\subsection{Evaluation}

We conducted a formative study to find out if TwisterSearch is capable of meeting our assumed design goals for collaborative Web searches and if it mediates during pre-, during-, and post-search activities. This includes general and situated

\footnotetext{
${ }^{2}$ Open Sound Control (OSC) - http://opensoundcontrol.org/

${ }^{3}$ Awesomium is a web-ui bridge for native apps - http://awesomium.com/

${ }^{4} \mathrm{Base} \mathrm{X}$ is a light-weight XML database - http://basex.org
} 


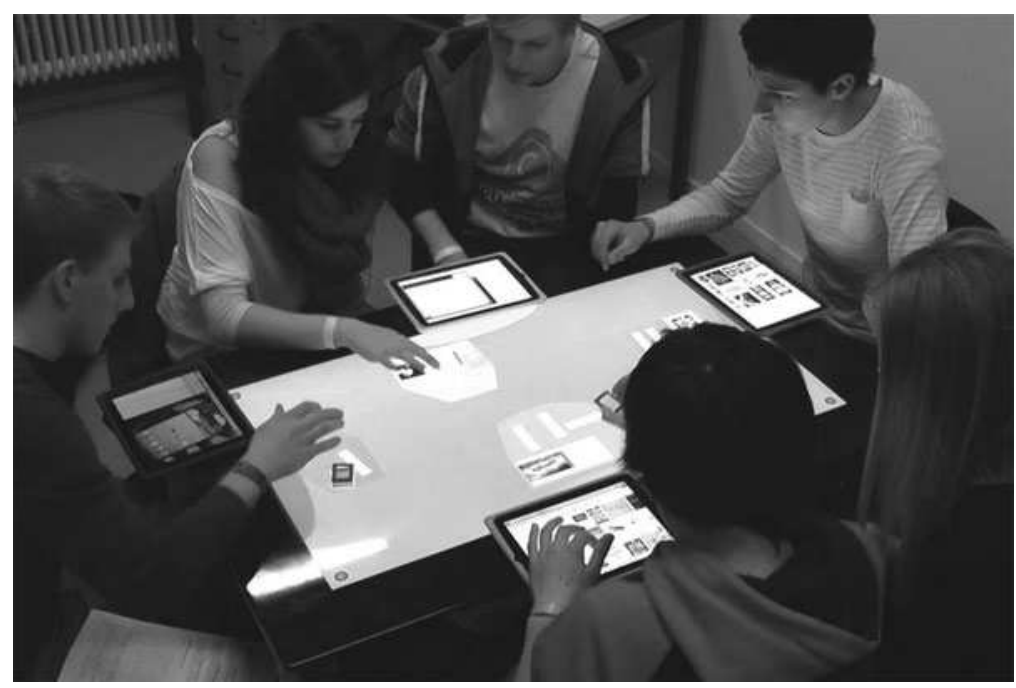

Fig. 5.7 Participants of the study searching the Web for evidence files. Each participant is wearing two bracelets (right and left wrist) according to their assigned TwisterSearch color. In this group, $2 \times 2$ participants had to share an iPad since the group consisted of six people and the prototype is equipped with four private displays only. The bracelets of the sharing partners have the same color but can be distinguished as one has plain colored bracelets and the other has colored bracelets with black stripes

awareness of the actions of co-workers and the applicability of TwisterSearch to the canonical model of social searches. Moreover, we wanted to identify barriers, "where the user must stop and learn many new concepts and techniques to make further progress" [11]. Therefore, we tested TwisterSearch in an educational setting and conducted the study in a high school with five groups in five different school subjects. Each subject was taught by a different teacher. We provided the teachers with one Samsung SUR40, four iPads, and both TwisterSearch applications for the table as well as for the iPads. The only requirement given to the teachers was that they had to prepare a lesson that demanded group work.

The tasks originate from the regular curriculum and were defined by the teachers themselves. Moreover, the teachers were not introduced to the technology beforehand; thus, the contents of teaching were made independently to allow us to test the general applicability of TwisterSearch to different teaching styles. Also, we did not want to purport assignment of tasks and thus avoid artificial tasks in advance.

The study generated data from pre- and post-test questionnaires (demographical, technical background), video recordings, interviews, and observations. During the study, the participants had to wear bracelets according to their assigned TwisterSearch color to distinguish the participants in the later video analysis (Fig. 5.7). The participants were asked to think aloud if they faced any problems or if they thought an important function was missing. 


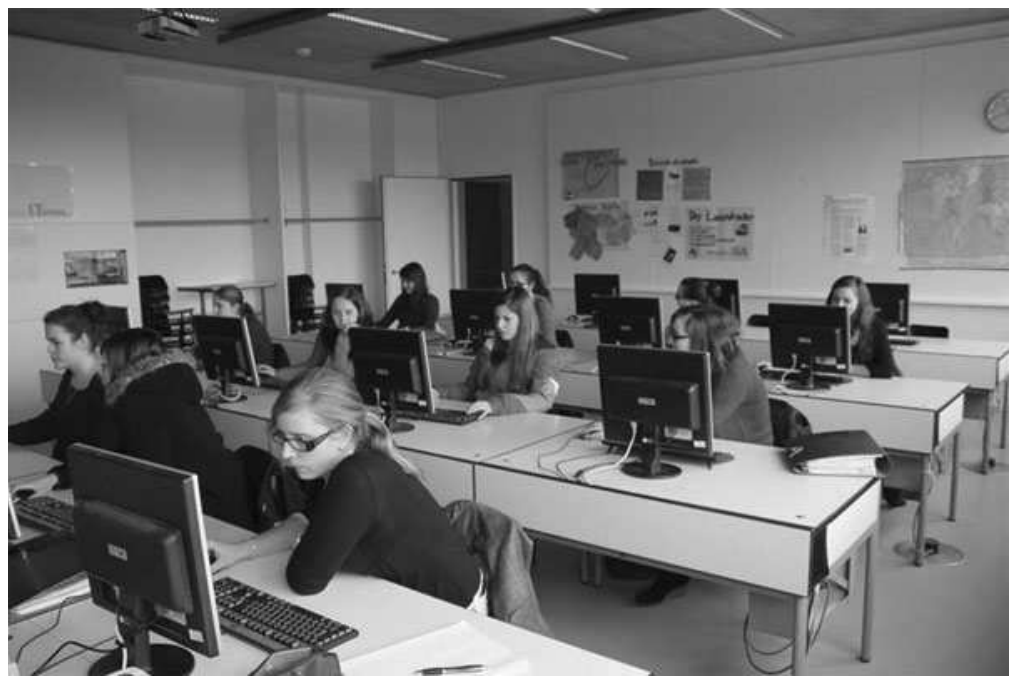

Fig. 5.8 The computer room with classmates performing the same task in groups of the same size. The door in the back connects to the observation room

A group consisted of 4-6 students, which result in an overall count of 23 students (20 male, 3 female). The mean age is 17.7 years $(\mathrm{SD}=1.07, \min =16, \max =20)$. Their mean computer experience in years is $9.65(\mathrm{SD}=1.95, \min =7, \max =14)$ and all participants had prior experience with touch-sensitive displays (e.g. smartphone, ATM, ticket machine, etc.). The mean value of their self-assessment of computer affinity is $3.04(\mathrm{SD}=0.69$, $\min =2)$, ranging from $1=$ beginner to $5=$ expert. The daily usage of computers is $\mathrm{M}=2.0(\mathrm{SD}=0.88)$ whereas the scale is $1=$ ' $\leq 1 \mathrm{~h}$ ', $2=$ ' 1 to $\leq 2 \mathrm{~h}$ ', $3=$ ' 2 to $\leq 3 \mathrm{~h}$ ', and $4=$ ' $>3 \mathrm{~h}$.'

The school subjects included in the study were Business Studies, Global Studies, Financial Management, German, and Geography. One group of each class was selected to perform their task with TwisterSearch while the other students had to use conventional desktop computers as illustrated in Fig. 5.8.

During the tasks, the test supervisor was present simply to provide assistance if technical issues occurred or usability issues hindered students in continuing their work since the study was meant to simulate an in "real life" setting. The teachers, however, were still allowed to help whenever task related questions arose.

Each group had a double period $(90 \mathrm{~min})$ to fulfill the given task, excluding time for technical instruction regarding the system as well as the training phase. Each function of the prototype was explained at first without pointing the students to the specific procedure that was derived from the canonical model of social searches. Afterwards, the students had enough time to "play" with TwisterSearch before doing the task and until they felt confident enough to operate the system. 


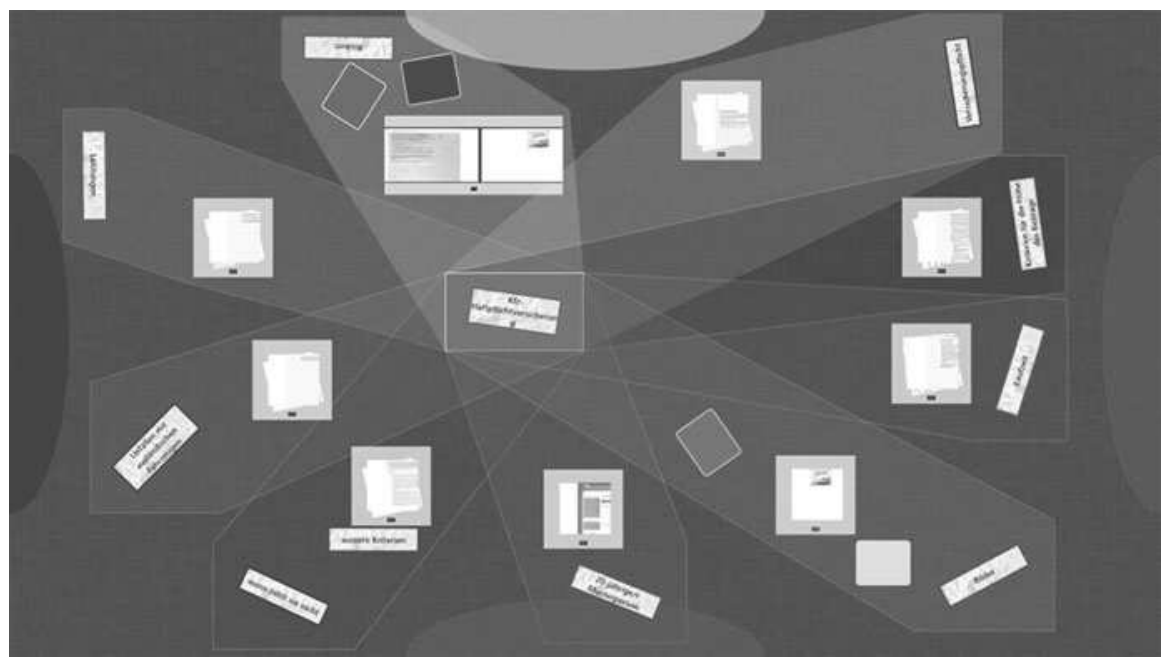

Fig. 5.9 The group followed the intrinsic procedure implemented in TwisterSearch, which is based on the canonical model of social searches

\subsection{Results}

The final outcomes of groups' Web search sessions differ in several aspects. Of course, the outcome depends on the task but also on the strategy a group chose to solve the task. In the following, we picked three final outcomes of Web search sessions to present two working styles that occurred most frequently during the study and one case were TwisterSearch was used for purposes other than originally intended (Figs. 5.9, 5.10, and 5.11).

Although, none of the groups were forced to follow the intended process of TwisterSearch, this group immediately started by brainstorming and collected keywords they thought of to be relevant to fulfill the task. Their task was to search for different qualities of vehicle insurance and provide a basic set of dimensions to qualitatively compare these insurance policies (e.g., compulsory insurance or liability in the event of damage). They generated the main topic 'vehicle insurance' in the center and started to diffuse in eight dimensions (clusters, see Fig. 5.9). After all members agreed on the skeleton of keywords, the group assigned the individual dimensions to single group members. Their approach confirms to the presearch activity and DG1 and therefore avoids 'Google races.' The Web search was performed solitary by the individual group members. After a group member was satisfied with the results, that person's responsibility changed to the next cluster. At the end of the search, participants put all tablet tokens into a single cluster and the person responsible for that cluster explained the contents. Other group members placed comprehensive questions, if necessary. However, the group did not 


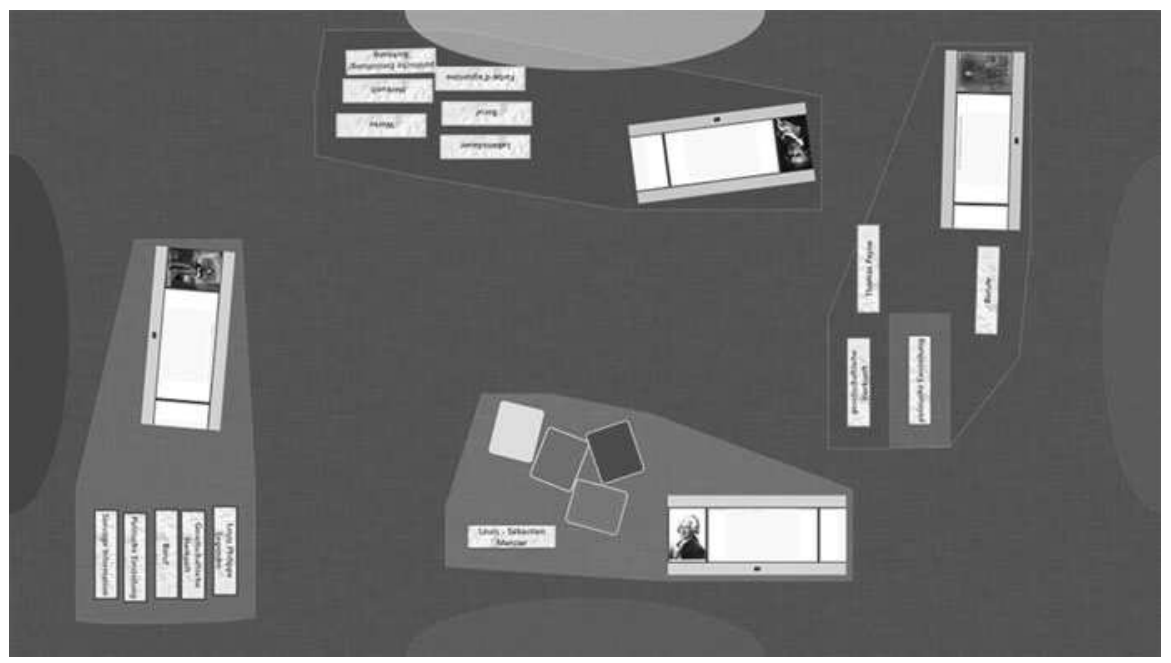

Fig. 5.10 A final outcome without prior brainstorming

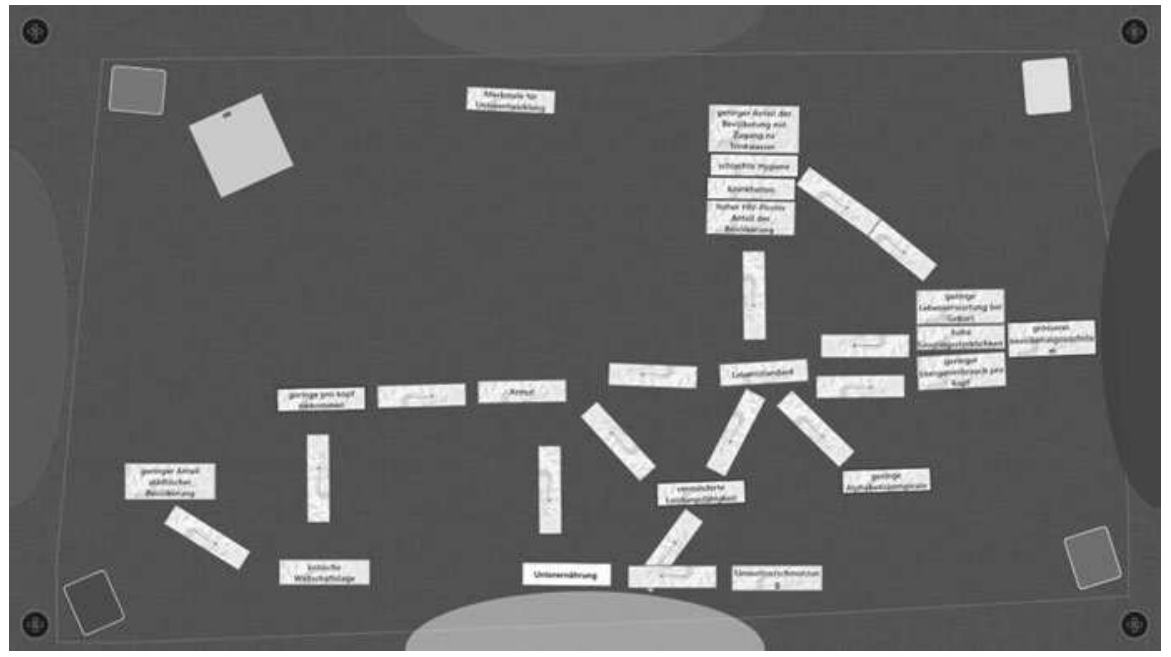

Fig. 5.11 The final outcome of a group that used TwisterSearch as a mind mapping tool

discuss or argue for or against certain results. The study did not clarify whether all participants were simply satisfied with the results or if TwisterSearch provides insufficient support for such discussion (DG3).

Another group started without brainstorming. Their task was to create a fact file for famous personalities (e.g., Louis Philippe Legendre or Thomas Paine). Beforehand, the teacher assigned each student with a known personality and instructed them to explain and discuss the facts once they were finished with 
their search. This led to four unique clusters in which each participant collected keywords individually (Fig. 5.10). However, the group exchanged valuable Web sources through face-to-face communication during the search. Again, this group did not discuss the results despite the fact that they explained the facts they found to other group members after the search. This resembles previous observations. Future studies should address this in more detail.

The third outcome illustrated in Fig. 5.11 is noteworthy since this group did not collect any Web resources. Instead they used TwisterSearch as a mind map tool to visualize the impact factors of standards of living. Of course, TwisterSearch was not implemented to be used as a mind mapping tool initially because of the missing functionality needed to create links between keywords. The group, however, managed to link words by creating keywords with ASCII-links (e.g., uni-directional links like "—_ $>$ "). The private devices were only used to create keywords and to obtain keywords by searching on task-related Web sites.

All participants were instructed to report on any problem and missing features during the study, which was jotted down by the investigator. For instance, two groups wanted to send links to websites without sharing them as a result. They requested it in order to start searching from the same informational base (other than 'Google'). The participants bypassed this by directing their team members to the website manually. Likewise, participants wanted to send selected text as keyword from the browser to the shared space instead of typing it manually. At the current stage of the prototype, it is impossible to alter the keyword text. The participants had to delete the keyword first and add a keyword with the corrected text thereafter. One group asked to be able to drag and drop the results from the result view on the landscape to freely arrange result items in clusters. Groups with a good number of keywords had an often cluttered landscape and several attempts by different participants were made to move entire clusters together with all keyword. Such requests to pan the landscape could imply the future integration of a zoomable user interface (ZUI) like ZOIL [12].

The qualitative feedback obtained through interviews indicates a better received quality of group work when asked for differences between regular group work and group work mediated by TwisterSearch. For instance, a participant mentioned that "Everyone can contribute to the work in an equal manner," which confirms DG2. Moreover, all participants reported having better awareness of the current and ongoing situation both for search responsibilities as well as completeness of the overall task. For instance, one participant argued that, "It is immediately apparent what the others are doing." However, the participant also criticized the longer period of vocational adjustment.

In addition to the study, we demonstrated TwisterSearch to teachers who were not taking part at the current study. These teachers were attracted by the didactic opportunities such a technology can provide. One teacher even mentioned a didactic method called Metaplan, ${ }^{5}$ which follows similar principles as those that

${ }^{5}$ http://en.wikipedia.org/wiki/Metaplan 
TwisterSearch incorporates. First, Metaplan requires a brainstorming phase where participants (a group of students) collect keywords on index cards and pin them on cork board. Of course, the collected keywords must match to the given task and are continuously clustered and augmented with strokes and arrows to express a common visual mind map. In some cases, teachers take a digital picture of the cork board and hand it out to students or students have to write down the mind map manually. With this as a basis, the group starts searching the Web for relevant information and assigns them directly to keywords on the visual mind map. The result of such a Metaplan session could be a poster or presentation.

This is very interesting for our research, as well-known didactic practices can be used in school subjects without necessarily changing the methodology to incorporate computer assistance.

The students in the computer room as illustrated in Fig. 5.8 are equipped with one personal computer each and groups were either seated in rows or blocks of $2 \times 2$ (two computers in a row and two rows). They were allowed to use tools of their choice to solve the task (e.g., Internet Explorer, etc.) and although exchange drives are provided by the school, most students used Facebook as platform in which to share teaching materials with their group members. This social platform allowed students to communicate with each other without yelling at each other in the classroom. However, they had to leave their workplace if face-to-face communication was required to show and highlight important aspects on a website.

\subsection{Conclusion and Future Work}

Based on the canonical model of social search by Evans and Chi [9] and implications for design described by Morris [7], we identified four design goals for the emerging topic of DUIs for collaborative search: (1). Support Strategic Planning and Coordination, (2). Amplify Collaboration, (3). Intensify Discussion and Simplify User Input, and (4). Facilitate Traceability of Evidence Files. On this basis, we presented the design and implementation of our interactive prototype TwisterSearch, which uses post-WIMP interaction with a tabletop computers and tablets to distribute collaborative Web searches across device boundaries. In a next step, we evaluated to what extent our design meets the design goals and enables efficient collaborative Web searches for educational purposes. Therefore, we conducted a qualitative user study similar to WeSearch [5]. After this, we will make TwisterSearch accessible to a broader user population in the library of the University of Konstanz to recruit participants for a controlled experiment with real library users and students in our lab.

Acknowledgments This work was partially supported by DFG Research Training Group GK-1042 "Explorative Analysis and Visualization of Large Information Spaces," University of Konstanz, and by the Ministry for Science, Research, and Art Baden-Wurttemberg under 
the project Blended Library. ${ }^{6}$ We especially thank Thomas Rädle and his colleagues at LES Sigmaringen for providing us the opportunity to evaluate and embed TwisterSearch in current classes, which served as an "real life" setting far beyond what controlled experiments and laboratories could offer.

\section{References}

1. Amershi, S., \& Morris, M. R. (2008). CoSearch: A system for co-located collaborative web search. Proceedings of the Twenty-Sixth Annual Sigchi Conference on Human Factors in Computing Systems - Proc. CHI 2008 (pp. 1647-1656).

2. Heilig, M. et al. (2011). Hidden details of negotiation: The mechanics of reality-based collaboration in information seeking. Proceedings of the 13th IFIP TC 13 International Conference on Human-Computer Interaction - Volume Part II (INTERACT'11) (pp. 622-639). Springer-Verlag, Berlin/Heidelberg.

3. Isenberg, P., \& Fisher, D. (2009). Collaborative brushing and linking for co-located visual analytics of document collections. Computer Graphics Forum, 28(3), 1031-1038.

4. Jetter, H. C. et al. (2011). Materializing the query with facet-streams - A hybrid surface for collaborative search on tabletops. Proceedings of the 2011 Annual Conference on Human Factors in Computing Systems (CHI'11) (pp. 3013-3022). ACM, New York.

5. Morris, M. R. et al. (2010). WeSearch: Supporting collaborative search and sensemaking on a tabletop display. Proceedings of the 2010 ACM Conference on Computer Supported Cooperative Work-CSCW'10 (pp. 401-410). New York: ACM Press.

6. Paul, S., \& Morris, M. (2009). CoSense: Enhancing sensemaking for collaborative web search. Proceedings of the SIGCHI Conference on Human Factors in Computing Systems (CHI'09) (pp. 1771). ACM Press, New York.

7. Morris, M. R. (2008). A survey of collaborative web search practices. Proceeding of the Twenty-Sixth Annual CHI Conference on Human Factors in Computing Systems - CHI'08. 1657.

8. Elmqvist, N. (2011). Distributed user interfaces: State of the art. In J. A. Gallud et al. (Eds.), Distributed user interfaces: Designing interfaces for the distributed ecosystem (pp. 1-12). London: Springer.

9. Evans, B. M., \& Chi, E. H. (2010). An elaborated model of social search. Information Processing \& Management, 46(6), 656-678.

10. Geyer, F. et al. (2011). Designing reality-based interfaces for creative group work. Proceedings of the 8th ACM Conference on Creativity and Cognition - C\&C'11 (p. 165). ACM Press, New York.

11. Myers, B., et al. (2000). Past, present, and future of user interface software tools. ACM Transactions on Computer-Human Interaction (TOCHI), 7(1), 3-28.

12. Zöllner, M., et al. (2011). ZOIL: A design paradigm and software framework for post-WIMP distributed user inter-faces. In J. A. Gallud et al. (Eds.), Distributed user interfaces: Designing interfaces for the distributed ecosystem (pp. 87-94). London: Springer.

${ }^{6}$ http://hci.uni-konstanz.de/blendedlibrary/ 